\title{
Lossless Positron Injection into a Magnetic Dipole Trap
}

\author{
E. V. Stenson, ${ }^{1,2,3, *}$ S. Nißl, ${ }^{1,2}$ U. Hergenhahn, ${ }^{1,4}$ J. Horn-Stanja, ${ }^{1}$ M. Singer, ${ }^{2}$ H. Saitoh, ${ }^{1,5}$ T. \\ Sunn Pedersen, ${ }^{1,6}$ J. R. Danielson, ${ }^{3}$ M. R. Stoneking, ${ }^{7}$ M. Dickmann, ${ }^{2}$ and C. Hugenschmidt ${ }^{2}$ \\ ${ }^{1}$ Max Planck Institute for Plasma Physics, Garching $\&$ Greifswald, Germany \\ ${ }^{2}$ Technische Universität München, Garching, Germany \\ ${ }^{3}$ University of California, San Diego, La Jolla, CA, U.S.A. \\ ${ }^{4}$ Leibniz Institute of Surface Engineering (IOM), Leipzig, Germany \\ ${ }^{5}$ The University of Tokyo, Kashiwa, Japan \\ ${ }^{6}$ University of Greifswald, Greifswald, Germany \\ ${ }^{7}$ Lawrence University, Appleton, WI, U.S.A.
}

(Dated: August 8, 2018)

\begin{abstract}
The high-efficiency injection of a low-energy positron beam into the confinement volume of a magnetic dipole has been demonstrated experimentally. This was accomplished by tailoring the three-dimensional guiding-center drift orbits of positrons via optimization of electrostatic potentials applied to electrodes at the edge of the trap, thereby producing localized and essentially lossless cross-field particle transport by means of the $\mathbf{E} \times \mathbf{B}$ drift. The experimental findings are reproduced and elucidated by numerical simulations, enabling a comprehensive understanding of the process. These results answer key questions and establish methods for use in upcoming experiments to create an electron-positron plasma in a levitated dipole device.
\end{abstract}

The nucleation and trapping of small-Debye-length electron-positron plasmas would enable novel laboratory studies of pair plasmas, which dominated the early universe during the lepton epoch (1 to $10 \mathrm{~s}$ after the Big Bang), are still found in astrophysical settings, and are predicted to have properties significantly different from those of standard electron-ion plasmas [1-6]. A fundamental challenge is the development of a scheme that allows efficient injection and subsequent confinement of both species. Injection and trapping of particles with a single sign of charge is well established in linear traps, which are used, for example, in the mass determination of unstable nuclei [7], in solvation studies of ionic species in water clusters [8], and in precision measurements of antimatter [9]. While linear traps can contain positrons and electrons simultaneously [10, 11], this has not yet been demonstrated with both species achieving plasma densities in the same volume. Toroidal traps with closed magnetic field lines, such as the stellarator $[12,13]$ and the levitated dipole [14-16], more readily confine positive and negative charges simultaneously, but injection from outside these devices is inhibited by the same physics that traps particles inside; electron-ion plasmas are typically ionized within the confinement region. Inward transport into a dipole due to turbulent interactions at high particle densities has been demonstrated for an electron beam [17]; however, typical particle densities of positron beams are well below the threshold for such collective interactions [18]. Additionally, the relatively small margin between the available flux of positrons and the amount needed to nucleate a plasma [19] necessitates that manipulation techniques for positrons strictly minimize losses. Therefore, electron-positron plasma experiments involving toroidal devices [19-21] require that efficient injection methods be developed and verified.
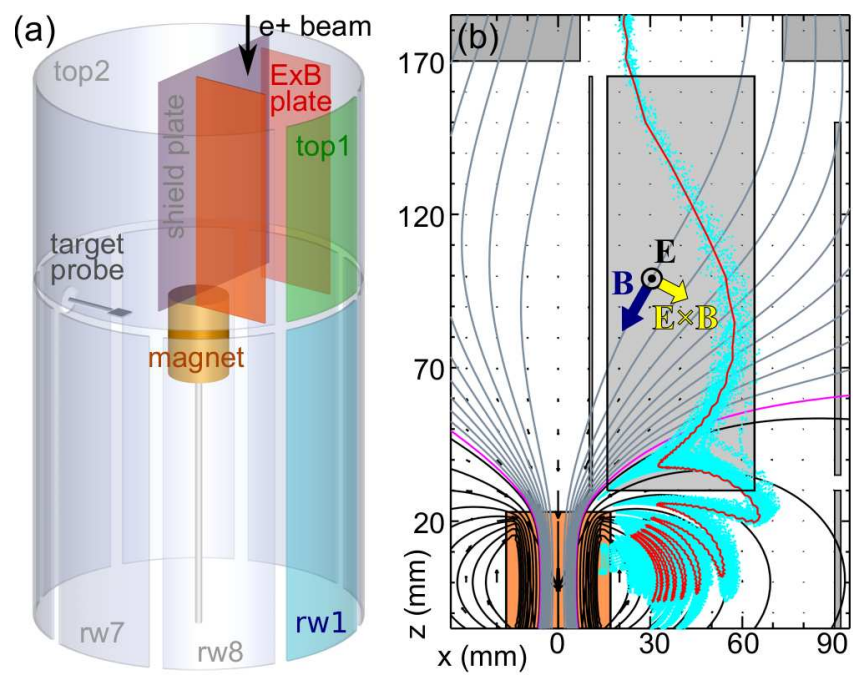

FIG. 1. a.) The experiment set-up. b.) A typical simulation of lossless $\mathbf{E} \times \mathbf{B}$ transport from field lines connecting to the beam line (top), across wall-limited magnetic field lines (including the separatrix, magenta), and into the confinement region. Points along the trajectories of 100 particles - launched from the same starting point with a realistic range of velocities and propagated for $0.6 \mu \mathrm{s}$ - are projected onto the $x z$ plane (cyan dots), as is a single trajectory with values of $E_{\|}$ and $E_{\perp}$ near the median of the distribution (red line).

Earlier numerical studies indicated that $\mathbf{E} \times \mathbf{B}$ drifts can transport particles into and out of a stellarator [22]. Experimental demonstration of positron injection into a prototype dipole trap was recently achieved with $38 \%$ efficiency, as was subsequent trapping [23]. We now report essentially lossless injection into that same device (Fig. 1), with a detailed analysis based on numerical simulations of both these and earlier experiments. This is a significant step towards studies of confined electron- 
positron plasmas, which we seek to realize by accumulating orders of magnitude more positrons into pulses [24], then injecting these (and electrons) into a levitated dipole trap [25].

As shown in Fig. 1a, the confining magnetic field is generated by a neodymium permanent magnet $(0.6 \mathrm{~T}$ at its poles), housed in a copper case and mounted with its dipole moment oriented vertically [23]. The outer wall, which has been divided into eight lower sections $(1 / 8$ each) and two upper sections $(1 / 8$ and $7 / 8)$, acts as a limiter, determining the size of the confinement region. (In a levitated dipole trap, the confinement region comprises closed field lines not touching any material objects; in the prototype trap, it comprises field lines intersecting only the magnet.) Low-energy charged particle motion in the trap is well described by guiding center theory [26, 27]. The center of the cyclotron orbit moves back and forth along the magnetic field with the effective potential energy $U=q \phi+\mu B$, where $\mu \equiv m v_{\perp}^{2} /(2 B)$ is an adiabatic invariant (with $q, m$ and $v_{\perp}$ being particle charge, mass, and velocity perpendicular to the magnetic field $\mathbf{B} ; \phi$ being the electrostatic potential; and $B=|\mathbf{B}|$ ). Additionally, the guiding center drifts across the magnetic field at the rate $v_{g c \perp}=\mathbf{B} \times \nabla U /\left(q B^{2}\right)+m v_{\|}^{2} \mathbf{B} \times \nabla B /\left(q B^{3}\right)$. Confined orbits consist of periodic motion at three wellseparated times scales: cyclotron orbits, magnetic mirroring, and slower toroidal drift around the magnet i.e., $80 \mathrm{MHz}-4 \mathrm{GHz}, 1-10 \mathrm{MHz}$, and 1-100 kHz, respectively, for $5-\mathrm{eV}$ positrons in a grounded trap $(\phi=0)$. The challenge is to transport particles onto these trapped orbits from an external source; this is accomplished by tailoring $U(\mathbf{r})$ via optimization of $\phi(\mathbf{r})$ (where $\mathbf{r}$ is spatial position).

The NEutron-induced POsitron source MUniCh (NEPOMUC), operated at the Research Neutron Source Heinz Maier-Leibnitz (FRM II), produces a nearly monoenergetic, high-brightness beam with an energy of $\sim 5$ $\mathrm{eV}$, a temperature of $\sim 2 \mathrm{eV}$, a spatial extent of $\sim 3 \mathrm{~mm}$ (FWHM), and a typical flux of $\sim 2 \times 10^{7}$ positrons $/ \mathrm{s}$ (3 pA) [28-30]. A magnetic guiding field (5 mT) transports the positrons to the dipole trap, installed at the open beam port and mated to the beam line by a set of Helmholtz coils. Positrons enter the experiment from above, along field lines connecting to the top of the magnet; the magnet's axis is parallel to but not coaxial with the Helmholtz coils'. On either side of these field lines are rectangular plates biased to $\pm V_{E \times B}$, creating an electric field mainly in the $y$ direction; this leads to the positrons drifting across the magnetic field into the confinement region (as shown in Fig. 1b), due to the $\mathbf{E} \times \mathbf{B}$ drift (the contribution to $v_{g c \perp}$ from $\mathbf{B} \times \nabla \phi$ ). A third plate, grounded and installed above the magnet, perpendicular to the $\mathbf{E} \times \mathbf{B}$ plates, shields the far side of the trap from the injection potential. A $1-\mathrm{cm} \times 1-\mathrm{cm}$ target probe (stainless steel) can be inserted in the equatorial plane of the trap on the opposite side from the injection re- gion, so as to determine the fraction of the beam that has been successfully injected into the confinement region and trapped for a half toroidal transit. The current to the target probe is measured with a charge-integrating amplifier and compared to the full beam current. The $\gamma$-radiation from positrons annihilating on the target is measured with a collimated scintillation detector (BGO). Further details can be found in the Supplemental Material [31].

A number of experimental parameters can be varied in order to optimize the injection of positrons into the trap. These include the bias used to produce the crossfield drift $\left(V_{E \times B}\right)$, the biases applied to the magnet case $\left(V_{m a g}\right)$ and the wall electrodes $\left(V_{t o p 1}, V_{t o p 2}\right.$, and $V_{r w 1}$ through $V_{r w 8}$; see Fig. 1a), and the currents in the steering coils $\left(I_{r}, I_{\theta}\right)$ that adjust the position of the beam right before it enters the main chamber of the experiment. Together these constitute a many-dimensional parameter space, which can be investigated via series of scans, such as the ones shown in Fig. 2a-b. Using this approach, we found conditions that result in the entire positron beam reaching the target probe. This occurs when $V_{t o p 1}, V_{r w 1}$, and $V_{\text {mag }}$ are set to positive biases greater than the beam energy, the rest of the outer electrode is grounded, and the appropriate $I_{r}, I_{\theta}$, and $V_{E \times B}$ are determined via pa-



FIG. 2. 2D scans such as these in $I_{r} V_{E \times B}$ were used to experimentally optimize injection conditions into the dipole trap. When biases are applied to electrodes rw1 and top1, and the rest of the wall was grounded (a), high-efficiency injection up to $100 \%$ - is achieved over a large region of the parameter space; this is not possible when all wall electrodes are biased (b). Single-particle simulations have been used to generate a "synthetic diagnostic" that produces good agreement with experiments $(\mathrm{c}, \mathrm{d})$. In all panels, the color scale indicates the fraction of the beam that reaches the target probe. 




FIG. 3. Spatial profile measurements are shown for lossless injection and for injection with the constraint that the entire outer wall be set to the same bias. Counts detected by the scintillator ( $\times$ and + , left axis; "probe out" value subtracted) and positron flux (solid symbols, right axis; measured with the charge-integrating amplifier) are each plotted as a function of target probe position and are in good agreement, as are synthetic profile measurements from simulations (open symbols). The black line and grey area indicate the average and standard deviation of measurements of total beam flux immediately upstream of the experiment; these and the vertical scatter of identical symbols illustrate typical uncertainties.

rameter scans. If instead the entire outer electrode is biased, the optimization procedure yields a maximum efficiency of $\sim 40 \%$ (Fig. 2b), reproducing our earlier work done with an unsegmented outer electrode [23]. Measurements at different target probe positions indicate where in the confinement region injected positrons are located (Fig. 3); these are by nature integrated spatial profiles, example derivatives of which are plotted in the Supplemental Information [31]. Systematic studies of how the different injection parameters can be used to influence the mean, width, and height of these profiles have been done and will be detailed in a separate publication.

Because the peak in Fig. 2a is wide in both $V_{E \times B}$ and $I_{r}$, we expect to be able to find efficient injection conditions for beams with larger energy spreads and/or larger spatial spreads. (The optimal setting of $V_{E \times B}$ is closely related to the energy of the injected beam; positrons with more kinetic energy, which move faster along the magnetic field lines and therefore spend less time between the parallel plates, require more $\mathbf{E} \times \mathbf{B}$ bias to be drifted by the same amount.) Significantly, these large tolerances for high injection efficiencies are found only when sufficient bias is applied to the top1 and rw1 electrodes. When either or both of those electrodes is grounded, not only is the peak injection efficiency lower, but the region of parameter space in which injection is achievable is severely restricted (Fig. 4). Biasing top2 (instead of grounding it) also reduces the tolerances in parameter space, though this is a comparatively mild effect, and lossless injection can still be achieved.
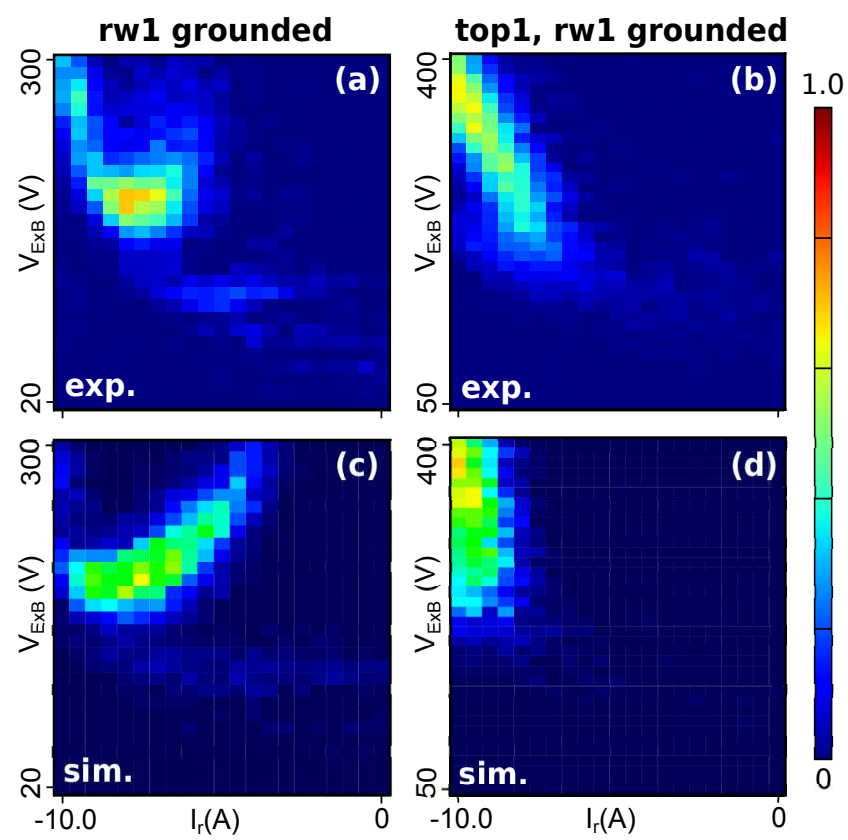

FIG. 4. Other configurations for injection - e.g., (a) grounding rw1 (while leaving the magnet and top1 biased) or (b) grounding the entire trap (except the $\mathbf{E} \times \mathbf{B}$ plates) - are possible but are less efficient and much more localized in parameter space. As in Fig. 2, the synthetic diagnostic (c,d) produces good qualitative and quantitative agreement.

So as to enable analyses of loss channels and their elimination, as well as separate examination of the injection and toroidal drift processes, simulations of the positron trajectories have been done both with the SIMION software package $[32,33]$ and with AlGeoJ, a home-grown particle mover that implements a modified leapfrog algorithm in Java [34]. Initial conditions can be generated as random draws from energy and spatial distributions matching those measured for the positron beam [30]. Synthetic diagnostics of the simulations produce both current and count "data" that can then be compared to experimental measurements, with the initial position of the beam center as an adjustable parameter. Further details can be found in the Supplemental Material [31], as well as in other publications [33, 34]. Injection efficiencies are reproduced in simulations using voltage and current settings identical to those used in the experiments.

Individual inspection of particle trajectories illuminates the physics behind the optimal parameter settings that result in lossless injection into the trap and subsequent toroidal transport to the target probe. Positrons are clearly on trapped particle orbits as they drift around the magnet and are particularly well-confined when wall biases are localized to the injection area (Fig. 5a). Simulations of our earlier experiments [23, 33] revealed the two primary loss channels that limited injection in that configuration to $\sim 40 \%$. First, there was a large loss cone for particles that did not have sufficient perpendicular 

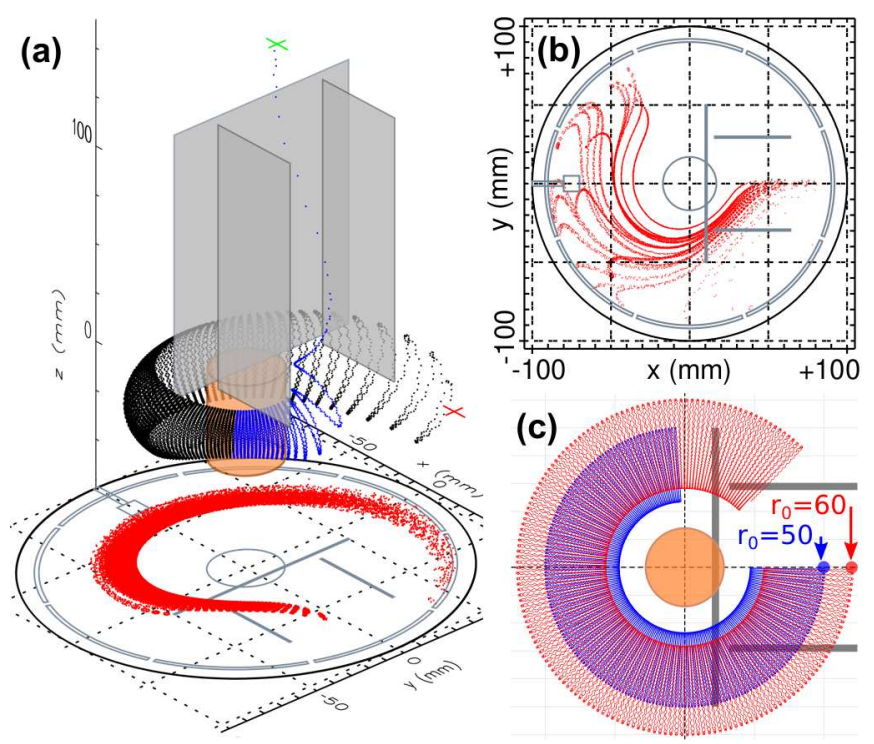

FIG. 5. Toroidal movement in the dipole trap was simulated for various cases of interest. (a) Lossless injection using localized biases. The entire trajectory for a representative particle is shown in blue (the first $0.6 \mu \mathrm{s}$ ) and black (thereafter); midplane crossings for the entire beam are shown in red, projected onto the bottom plane. (b) Mid-plane crossings for the entire beam, for conditions with the entire wall at $5.5 \mathrm{~V}$ and the magnet at $-15 \mathrm{~V}$. (c) Two particles in an ideal dipole trap (without electrostatic potentials or beam line magnetic fields), started with the same velocity vector but different radii.

velocity to magnetically mirror before hitting the negatively biased magnet case $(\sim 40 \%)$. Second, enhanced outward transport during the first half toroidal transit resulted in further losses $(\sim 20 \%)$, as shown in Fig. 5 b.

Particles are injected into a range of orbits (different radii from the magnet, different energies), due to the beam's finite spatial and energy spreads, resulting in different rates of toroidal drift (e.g., a particle with the same velocity vector at a smaller radius in a grounded dipole trap goes around more slowly than one at a larger radius, as shown in Fig. 5c). Thus, a particle bunch will spread out within a few toroidal orbits around the magnet. (Note that although particles are lost after a single orbit if $V_{E \times B}$ is applied steady state, switching off $V_{E \times B}$ after injection results in trapped particles with confinement times of hundreds [23] to tens of thousands [33, 35] of toroidal transits.)

As mentioned previously, injection that is both lossless and robust across the parameter space is achievable in the prototype trap when both rw1 and top1 are biased. To illustrate why this is, we examine a different plane in the parameter space for injection with top1 and top2 set to the same bias and, more significantly, rw1 grounded. In this case, injection is possible only for small islands in the parameter space (Fig. 6a) with lower efficiency than when rw1 is biased (Fig. 6b). The simulated diagnostic (Fig. 6c) reproduces the key features of the experimental


FIG. 6. The islands that are seen in the 2D optimization scans of the $V_{\text {top } 1 \& 2} V_{E \times B}$ parameter space plane when wall segment rw1 is grounded (a) - in contrast to when rw1 is biased above the beam energy (b) - were reproduced by the synthetic diagnostic (c). Examination of particle trajectories (d) shows that each island corresponds to particles entering the confinement region after a different number of electrostatic reflections off the biased upper wall segment. (The three different $V_{t o p 1 \& 2}$ and $V_{E \times B}$ settings used for the trajectory simulations shown in (d) - labeled 0,1 , and 2 for the number of reflections - are indicated by asterisks in (c).)

results, and particle trajectories (Fig.6d) demonstrate that successful injection does not necessarily occur via a single magnetic mirroring event (trajectory 0) but can also follow multiple mirroring events that alternate with electrostatic reflection off the outer wall (trajectories 1 , $2)$. When rw1 is grounded, trajectories that would otherwise have reflected off a biased rw1 to become trapped in the confinement region do not do so; tracking of particle "hit locations" confirms that annihilation on rw1 is indeed a primary loss channel [34].

All of the following are desirable: that injection efficiency be maximized, that the injection region be localized (to enable stacking of pulses, e.g.), and that the "sweet spot" in parameter space be large (to accommodate beams' spatial and energy spreads, as well as to give more options in terms of which beam settings can be used). There was no guarantee that these conditions could all be met simultaneously, but indeed this is the case. Therefore, $\mathbf{E} \times \mathbf{B}$ drift injection is a viable approach that can be incorporated into the design for the levitated dipole experiment [25]. There will be some key differ- 
ences from the prototype device (e.g., magnetic field lines of the confinement region will not intersect the magnet), for which the details of the injection scheme will need to be adjusted accordingly; simulations that can reproduce the experimental results in the prototype trap constitute a powerful tool for engineering efficient injection into the levitated trap. Finally, upcoming experiments will extend drift injection - used to date with a steadystate positron beam - for use with one or more pulses of positrons accumulated beforehand in linear traps [24], so as to achieve orders of magnitude more confined positrons in the dipole trap. Although the experiments reported here are in the single-particle regime, the technique can be expected to work well up to densities at which collective behavior of the positrons screens out the injection potentials - that is, near to the goal of achieving plasma densities [18, 22].

In summary, we have shown that a low-density, lowenergy positron beam can be injected with $\sim 100 \%$ efficiency into the confinement region of a magnetic dipole field. This was done by strategically tailoring the effective potential energy $U=e \phi+\mu B$ to produce the desired guiding center drifts for realistic beam properties. This finding is essential for plans to produce a confined electron-positron plasma in a laboratory magnetosphere [19-21]. As a further perspective, applying our scheme to trap molecular ions can be conceived; here, it could be used to study cation-anion reactions at low temperatures, which are only accessible with great effort otherwise [36].

This work is based upon experiments performed at the NEPOMUC positron beam facility operated by FRM II at the Heinz Maier-Leibnitz Zentrum (MLZ), Garching, Germany. The authors gratefully credit Sebastian Vohburger for his contribution to setting up the experiment. This work was supported by the European Research Council (ERC-2016-ADG No.741322), the Deutsche Forschungsgemeinschaft $(\mathrm{Hu}$ 978/15, Sa 2788/2), the Max Planck Institute for Plasma Physics, the NIFS Collaboration Research Program, Japan Society for the Promotion of Science (JSPS Grants-inAid for Scientific Research 25707043 and 16KK0094), the Helmholtz Association Postdoc Programme (E.V.S.), and the UCSD Foundation.

* E-Mail: evs@ipp.mpg.de

[1] T. Siegert, R. Diehl, G. Khachatryan, M. G. H. Krause, F. Guglielmetti, J. Greiner, A. W. Strong, and X. Zhang, "Gamma-ray spectroscopy of positron annihilation in the Milky Way," A\&A 586, A84 (2016).

[2] L. O. Silva, R. A. Fonseca, J. W. Tonge, J. M. Dawson, W. B. Mori, and M. V. Medvedev, "Interpenetrating plasma shells: Near-equipartition magnetic field generation and nonthermal particle acceleration," The Astrophysical Journal Letters 596, L121 (2003).
[3] V. Tsytovich and C. B. Wharton, "Laboratory electronpositron plasma - a new research object," Comments Plasma Phys. Controlled Fusion 4, 91-100 (1978).

[4] W. Oohara, D. Date, and R. Hatakeyama, "Electrostatic waves in a paired fullerene-ion plasma," Phys. Rev. Lett. 95, 175003 (2005).

[5] P. Helander, "Microstability of magnetically confined electron-positron plasmas," Phys. Rev. Lett. 113, 135003 (2014).

[6] G. Sarri, M. E. Dieckmann, I. Kourakis, A. Di Piazza, B. Reville, C. H. Keitel, and M. Zepf, "Overview of laserdriven generation of electron-positron beams," Journal of Plasma Physics 81 (2015), 10.1017/S002237781500046X.

[7] H. Stolzenberg, St. Becker, G. Bollen, F. Kern, H.-J. Kluge, Th. Otto, G. Savard, L. Schweikhard, G. Audi, and R. B. Moore, "Accurate mass determination of shortlived isotopes by a tandem penning-trap mass spectrometer," Phys. Rev. Lett. 65, 3104-3107 (1990).

[8] G. Niedner-Schatteburg and V. E. Bondybey, "FT-ICR studies of solvation effects in ionic water cluster reactions," Chemical Reviews 100, 4059-4086 (2000), pMID: 11749340, http://dx.doi.org/10.1021/cr990065o.

[9] M. Ahmadi, B. X. R. Alves, C. J. Baker, W. Bertsche, A. Capra, C. Carruth, C. L. Cesar, M. Charlton, S. Cohen, R. Collister, S. Eriksson, A. Evans, N. Evetts, J. Fajans, T. Friesen, M. C. Fujiwara, D. R. Gill, J. S. Hangst, W. N. Hardy, M. E. Hayden, C. A. Isaac, M. A. Johnson, J. M. Jones, S. A. Jones, S. Jonsell, A. Khramov, P. Knapp, L. Kurchaninov, N. Madsen, D. Maxwell, J. T. K. McKenna, S. Menary, T. Momose, J. J. Munich, K. Olchanski, A. Olin, P. Pusa, C. . Rasmussen, F. Robicheaux, R. L. Sacramento, M. Sameed, E. Sarid, D. M. Silveira, G. Stutter, C. So, T. D. Tharp, R. I. Thompson, D. P. van der Werf, and J. S. Wurtele, "Characterization of the 1S-2S transition in antihydrogen," Nature (2018).

[10] R. G. Greaves and C. M. Surko, "An electron-positron beam-plasma experiment," Phys. Rev. Lett. 75, 38463849 (1995).

[11] H. Higaki, C. Kaga, K. Fukushima, H. Okamoto, Y. Nagata, Y. Kanai, and Y. Yamazaki, "Simultaneous confinement of low-energy electrons and positrons in a compact magnetic mirror trap," New Journal of Physics 19, 023016 (2017)

[12] X. Sarasola and T. Sunn Pedersen, "First experimental studies of the physics of plasmas of arbitrary degree of neutrality," Plasma Physics and Controlled Fusion 54, 124008 (2012).

[13] P. Helander, "Theory of plasma confinement in nonaxisymmetric magnetic fields," Reports on Progress in Physics 77, 087001 (2014).

[14] A. Hasegawa, "A dipole field fusion reactor," Comments on Plasma Physics and Controlled Fusion 11, 147-151 (1987).

[15] D. T. Garnier, A. Hansen, M. E. Mauel, E. Ortiz, A. C. Boxer, J. Ellsworth, I. Karim, J. Kesner, S. Mahar, and A. Roach, "Production and study of high-beta plasma confined by a superconducting dipole magnet," Physics of Plasmas (1994-present) 13, 056111 (2006).

[16] M. Nishiura, Y. Kawazura, Z. Yoshida, N. Kenmochi, Y. Yano, H. Saitoh, M. Yamasaki, T. Mushiake, A. Kashyap, N. Takahashi, M. Nakatsuka, and A. Fukuyama, "Ion cyclotron resonance heating system in the RT-1 magnetospheric plasma," Nuclear Fusion 57, 086038 (2017). 
[17] Z. Yoshida, H. Saitoh, J. Morikawa, Y. Yano, S. Watanabe, and Y. Ogawa, "Magnetospheric vortex formation: Self-organized confinement of charged particles," Phys. Rev. Lett. 104, 235004 (2010).

[18] E. V. Stenson, J. Horn-Stanja, M. R. Stoneking, and T. Sunn Pedersen, "Debye length and plasma skin depth: two length scales of interest in the creation and diagnosis of laboratory pair plasmas," Journal of Plasma Physics 83 (2017), 10.1017/S0022377817000022.

[19] T. Sunn Pedersen, J. R. Danielson, C. Hugenschmidt, G. Marx, X. Sarasola, F. Schauer, L. Schweikhard, C. M. Surko, and E. Winkler, "Plans for the creation and studies of electron-positron plasmas in a stellarator," New Journal of Physics 14, 035010 (2012).

[20] H Saitoh, T S Pedersen, U Hergenhahn, E V Stenson, $\mathrm{N}$ Paschkowski, and C Hugenschmidt, "Recent status of A Positron-Electron eXperiment (APEX)," Journal of Physics: Conference Series 505, 012045 (2014).

[21] E. V. Stenson, H. Saitoh, J. Stanja, H. Niemann, U. Hergenhahn, T. Sunn Pedersen, G. H. Marx, L. Schweikhard, J. R. Danielson, C. M. Surko, and C. Hugenschmidt, "Progress toward positron-electron pair plasma experiments," AIP Conference Proceedings 1668, 040004 (2015), http://dx.doi.org/10.1063/1.4923117.

[22] B. D. de Gevigney, T. Sunn Pedersen, and A. H. Boozer, "Debye screening and injection of positrons across the magnetic surfaces of a pure electron plasma in a stellarator," Physics of Plasmas 18, 013508 (2011).

[23] H. Saitoh, J. Stanja, E. V. Stenson, U. Hergenhahn, H. Niemann, T. Sunn Pedersen, M. R. Stoneking, C. Piochacz, and C. Hugenschmidt, "Efficient injection of an intense positron beam into a dipole magnetic field," New Journal of Physics 17, 103038 (2015).

[24] J. R. Danielson, D. H. E. Dubin, R. G. Greaves, and C. M. Surko, "Plasma and trap-based techniques for science with positrons," Rev. Mod. Phys. 87, 247-306 (2015).

[25] M. R. Stoneking, H. Saitoh, M. Singer, E. V. Stenson, J. Horn-Stanja, T. Sunn Pedersen, S. Nißl, U. Hergenhahn, N. Yanagi, C. Hugenschmidt, M. Dickmann, J. R. Danielson, and C. M. Surko, "Toward a compact levitated superconducting dipole for positron-electron plasma confinement," AIP Conference Proceedings 1928, 020015 (2018), http://aip.scitation.org/doi/pdf/10.1063/1.5021580.

[26] R. J. Goldston and P. H. Rutherford, Introduction to Plasma Physics (CRC Press, 1995).

[27] A. H. Boozer, "Time-dependent drift Hamilto- nian," The Physics of Fluids 27, 2441-2445 (1984), https://aip.scitation.org/doi/pdf/10.1063/1.864525.

[28] C. Hugenschmidt, C. Piochacz, M. Reiner, and K. Schreckenbach, "The NEPOMUC upgrade and advanced positron beam experiments," New Journal of Physics 14, 055027 (2012).

[29] C. Hugenschmidt, H. Ceeh, T. Gigl, F. Lippert, C. Piochacz, M. Reiner, K. Schreckenbach, S. Vohburger, J. Weber, and S. Zimnik, "Positron beam characteristics at NEPOMUC upgrade," Journal of Physics: Conference Series 505, 012029 (2014).

[30] J. Stanja, U. Hergenhahn, H. Niemann, N. Paschkowski, T. Sunn Pedersen, H. Saitoh, E.V. Stenson, M.R. Stoneking, C. Hugenschmidt, and C. Piochacz, "Characterization of the NEPOMUC primary and remoderated positron beams at different energies," Nuclear Instruments and Methods in Physics Research Section A: Accelerators, Spectrometers, Detectors and Associated Equipment 827, 52 - 62 (2016).

[31] See Supplemental Material at [URL] for additional details of the experiment and simulation set-ups, as well as complementary presentations of select results.

[32] D. Manura and D. Dahl, SIMION (R) 8.0 User Manual, Scientific Instrument Services, Inc., Ringoes, NJ 08551 (2008).

[33] U. Hergenhahn, J. Horn-Stanja, S. Nißl, T. Sunn Pedersen, H. Saitoh, E. V. Stenson, M. R. Stoneking, M. Dickmann, C. Hugenschmidt, M. Singer, J. R. Danielson, and C. M. Surko, "Progress of the APEX experiment for creation of an electron-positron pair plasma," AIP Conference Proceedings 1928, 020004 (2018), http://aip.scitation.org/doi/pdf/10.1063/1.5021569.

[34] Stefan Nißl, Numerical Investigations into Injection and Confinement of Single Particles in a Magnetic Dipole Trap, Master's thesis, Technische Universität München (2018).

[35] J. Horn-Stanja et al., In preparation.

[36] R. D. Thomas, H. T. Schmidt, G. Andler, M. Bjrkhage, M. Blom, L. Brnnholm, E. Bckstrm, H. Danared, S. Das, N. Haag, P. Halldn, F. Hellberg, A. I. S. Holm, H. A. B. Johansson, A. Kllberg, G. Kllersj, M. Larsson, S. Leontein, L. Liljeby, P. Lfgren, B. Malm, S. Mannervik, M. Masuda, D. Misra, A. Orbn, A. Pal, P. Reinhed, K.-G. Rensfelt, S. Rosn, K. Schmidt, F. Seitz, A. Simonsson, J. Weimer, H. Zettergren, and H. Cederquist, "The double electrostatic ion ring experiment: A unique cryogenic electrostatic storage ring for merged ion-beams studies," Review of Scientific Instruments 82, 065112 (2011), https://doi.org/10.1063/1.3602928. 